\title{
Fobia alimentar associada a magreza: um diagnóstico diferencial com anorexia nervosa
}

\author{
Low weight associated with food phobia: a
}

differential diagnosis with anorexia nervosa

Vera Garcia da Silva', Marcelo Papelbaum

\section{RESUMO}

A fobia alimentar (FA) ou fagofobia é um transtorno caracterizado pelo medo condicionado e excessivo de comer e engolir, muitas vezes precipitado por um evento de vômito ou engasgo. Existem poucos casos de FA descritos na literatura científica, o que dificulta a definição da sua prevalência. Apesar disso, a fagofobia apresenta importância clínica em função do risco de complicações clínicas e da possibilidade de erro diagnóstico por confusão com outras condições que acarretem restrição alimentar, como a anorexia nervosa. O objetivo deste artigo é apresentar um caso de FA e discutir a psicopatologia desse transtorno, as dificuldades diagnósticas e recomendações terapêuticas, tendo como base a evolução clínica do caso e as evidências científicas atuais.

\section{Palavras-chave}

Comportamento alimentar, fagofobia, caso clínico, fobia alimentar.

\section{Keywords}

Eating behavior, phagophobia, case report, food phobia.

\begin{abstract}
Food phobia (FP) or phagophobia is a disorder characterized by an excessive conditioned fear of eating and swallowing, many times precipitated by vomiting or choking. There are few reports of FP on scientific literature, so it's difficult to know its prevalence. Despite of that, it presents clinical relevance because of the risk of clinical complications and misdiagnosis possibility by confusion with other conditions that carries food restraint, like anorexia nervosa. The objective of this article is to present a FP clinical case and to discuss the psychopathology, diagnostic difficulties and recommended therapeutics, based on clinical evolution of the case and recent scientific evidences.
\end{abstract}

\section{INTRODUÇÃO}

A fobia alimentar (FA), ou fagofobia, é caracterizada pelo medo excessivo e condicionado de comer ou engolir, levando à recusa alimentar por mais de um mês. Muitas vezes, é desencadeada após um evento traumático em que tenham ocorrido vômitos ou sensação de asfixia provocada pela comida. É mais comum o início na infância (principalmente em mulheres), podendo se iniciar após alguma infecção ou evento que tenha cursado com vômito associado. A vivência traumática desse episódio pode ocasionar um comportamento mal-adaptativo, como a recusa alimentar.

Nenhum dos sistemas classificatórios atuais (DSM e CID) inclui a FA como um diagnóstico independente. Sendo assim, ela poderia ser classificada com um transtorno alimentar não especificado ou uma fobia específica.

Higgs et al. (1989) apresentaram, num estudo retrospectivo e longitudinal, um grupo de crianças que teria um

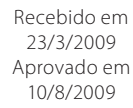


transtorno intermediário, entre a anorexia nervosa e evitação à comida, como um transtorno parcial de anorexia.

Em 1996, Kim et al. realizaram um estudo prospectivo de pacientes com disfagia de etiologias diversas e observaram que havia alta prevalência de transtornos psiquiátricos como depressão, ansiedade e somatizações, em pacientes com contrações esofagianas específicas, em comparação àqueles sem contrações esofagianas.

O termo "choking phobia" foi usado por alguns autores durante um período, mas não fazia distinção entre aqueles pacientes com disfagia psicogênica e aqueles que engasgavam ou aspiravam os alimentos por outras causas.

Em artigo de 1997, Shapiro et al. sugeriram o termo "fagofobia", para designar os casos de disfagia psicogênica, em que os pacientes apresentam queixas de dificuldade para engolir, com exame físico e achados laboratoriais normais. Esses pacientes comumente consultam vários especialistas, como gastroenterologistas, otorrinolaringologistas e neurologistas, antes de procurar o psiquiatra, muitas vezes com queixa de sensação de bolo na garganta. Os pacientes apresentam a sensação de serem incapazes de engolir o alimento, algumas vezes acompanhada de medo de aspirar. Como esses pacientes acreditam que têm uma doença orgânica, raramente procuram o psiquiatra. E o diagnóstico é feito tardiamente.

A prevalência da FA é desconhecida, sobretudo em função das dificuldades diagnósticas. De fato, é bastante frequente o erro diagnóstico por confusão com outros transtornos psiquiátricos, sobretudo com os transtornos alimentares, especialmente anorexia nervosa. Porém, na FA não ocorrem a preocupação com ganho de peso e a distorção da autoimagem corporal, mas, em função da perda de peso associada e da recusa alimentar, esses pacientes são comumente diagnosticados com anorexia nervosa.

A Figura 1 apresenta as características clínicas dos principais diagnósticos diferenciais psiquiátricos da fagofobia.

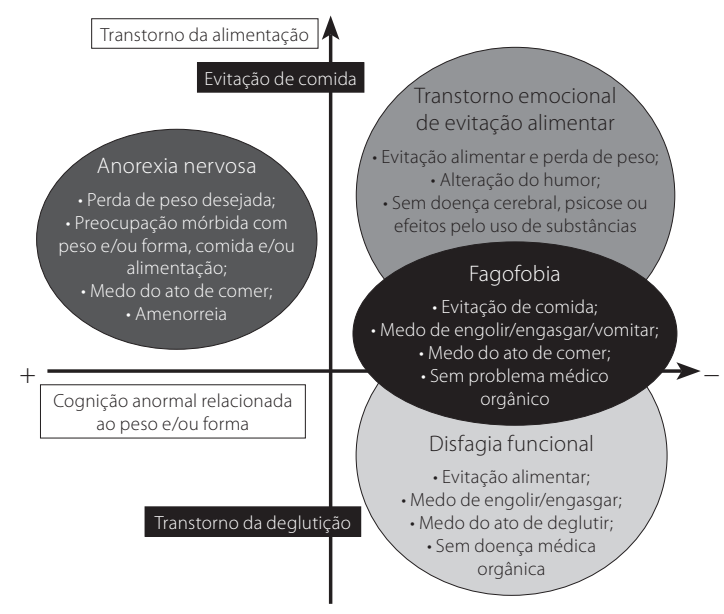

Figura traduzida e adaptada após a autorização de Okada et al. (2007).

Figura 1. Diagnósticos diferenciais da fagofobia.
Outros diagnósticos clínicos também poderiam dificultar o diagnóstico e devem ser excluídos, sobretudo quando queixas gastrointestinais são frequentes. Exames que avaliem a anatomia e funcionalidade do trato digestivo, sobretudo a endoscopia digestiva alta (EDA) e a esofagoscopia, podem ajudar a eliminar causas orgânicas como hérnias de hiato, hipotireoidismo ou tumores.

Apesar das dificuldades diagnósticas, a fagofobia deve ter sua avaliação e seu tratamento implementados o mais rápido possível, pois apresenta uma série de complicações clínicas possíveis, sobretudo as decorrentes de uma perda de peso rápida associada à restrição alimentar. Quando ocorrem vômitos, distúrbios hidroeletrolíticos e lesões gástricas e esofagianas também podem aparecer.

\section{CASO CLÍNICO}

História da doença atual: L. é uma jovem de 20 anos, solteira, natural do Rio de Janeiro e estudante do ensino médio. Foi encaminhada para uma avaliação psiquiátrica pelo endocrinologista por "problemas alimentares" e pesando 36,7 kg (índice de massa corporal de $15,27 \mathrm{~kg} / \mathrm{m}^{2}$ ). L. relata que, por volta dos 8 anos de idade, apresentou vômitos e diarreia por um dia, após uma refeição numa lanchonete de comida rápida. Desde então, começou a rejeitar certos alimentos e a restringir a sua ingestão alimentar. Sentia náuseas só de pensar no ato de comer. Apesar de não apresentar dificuldades para engolir, cuspia parte da comida, com medo de vomitar. Com o tempo, passou a se alimentar melhor, porém, se ocorresse qualquer problema que a deixasse mais ansiosa, voltava a restringir sua alimentação. Esse quadro se acentuou no último ano. A paciente passou a acordar durante a madrugada sentindose mal e temendo vomitar. Dormia com antieméticos embaixo do travesseiro e ficava nervosa e nauseada nos momentos que antecediam às refeições, sempre imaginando que algo poderia estar estragado. Em contrapartida, L. frequentemente ingeria uma grande quantidade de balas em tempo curto. Alimentava-se basicamente de arroz e bife feitos em casa e evitava todos os outros tipos de comida, especialmente em festas, quando temia passar mal na frente de outras pessoas. Sempre se considerou "anormal" por não comer como os outros e reconhecia a sua magreza, desejando o ganho de peso. Com o tempo, L. começou a se aproveitar de seus sintomas para punir seus pais, que a tratavam de forma repressora. Ao exame, todas as alterações psicopatológicas observadas pareciam secundárias ao medo de comer per si.

História pessoal: $L$. possui várias amigas, mas sua vida social é bastante limitada pelos seus pais. Segundo L., seu pai tem temperamento "forte" e evidencia história de dependência ao álcool prévia (abstinente desde 1995). Apesar dos conflitos familiares, L. teve muita dificuldade para se separar dos pais na ocasião em que ingressou na escola. 
Exames complementares: Avaliação laboratorial sanguínea (incluindo bioquímica e hemograma) e endoscopia digestiva alta normais.

Hipótese diagnóstica: Fobia alimentar (transtorno alimentar não especificado ou fobia específica pelo DSM-IV).

Diagnósticos diferenciais: $L$. não apresentava pensamentos obsessivos ou rituais além daqueles estritamente relacionados aos alimentos, não apresentando, portanto, sintomas sugestivos de transtorno obsessivo-compulsivo (TOC); também não se percebia gorda, ao contrário, sofria com sua magreza excessiva, e desejava engordar. Ficava bastante ansiosa nos momentos que precediam as refeições, mas comportava-se normalmente em outras ocasiões do seu cotidiano. Dessa forma, foram excluídos os diagnósticos de anorexia nervosa (AN), transtorno de ansiedade sem outra especificação e transtorno factício.

Tratamento: Foram iniciados psicoterapia individual e acompanhamentos nutricional e psiquiátrico.

Para um tratamento adequado, Okada et al. (2007) propõem classificar a FA em 2 tipos: pós-traumático e conversivo. Neste caso, a paciente apresenta um quadro misto.

O tratamento farmacológico, com o objetivo de redução das queixas ansiosas, foi realizado com o uso da clomipramina (até o máximo de $225 \mathrm{mg} / \mathrm{dia}$ ) e buspirona 10 mg/dia.

O acompanhamento psicológico tinha como meta implementar estratégias para melhorar a comunicação interpessoal e diminuir a ansiedade relacionada à alimentação. Além disso, foram realizadas sessões psicoeducativas com os pais de $L$.

O acompanhamento nutricional pretendia reduzir estigmas relacionados aos alimentos e planejar cardápios que a paciente conseguisse seguir e que garantissem as necessidades nutricionais diárias.

Evolução clínica: Com 1 mês de tratamento, houve ganho de $2 \mathrm{~kg}$ e melhora das queixas gastrointestinais. Aos 3 meses de acompanhamento, L. já se alimentava sem restrições. Ao longo de 10 meses de tratamento, houve redução significativa e sustentada da psicopatologia alimentar, além de um ganho ponderal de $8 \mathrm{~kg}$. Durante todo o período de tratamento, a paciente manteve acompanhamento endocrinológico. Posteriormente, a clomipramina foi reduzida até a dose de $150 \mathrm{mg}$ ao dia.

Atualmente, o tratamento consiste em clomipramina 50 $\mathrm{mg} / \mathrm{dia}$, psicoterapia de apoio e acompanhamento semestral com a endocrinologista.

Está cursando uma faculdade e namorando.

\section{DISCUSSÃO}

McNally propõe que a fagofobia é, muitas vezes, o resultado de uma experiência traumática vivida ou presenciada pelo paciente, envolvendo, por exemplo, engasgo com comida ou remédio.

No artigo de relato de caso, Ciyiltepe e Turkbay (2006) ressaltam que a fagofobia deve ser um diagnóstico de exclusão, e uma investigação clínica prévia deve ser realizada para afastar-se uma causa orgânica.

Apesar do risco de complicações clínicas, a FA não é considerada um transtorno psiquiátrico independente. Neste caso, a paciente apresentava ansiedade antecipatória ao objeto temido e à comida, e comportamento de esquiva associado. Entretanto, a psicopatologia apresentada era exclusivamente relacionada ao comportamento alimentar e às complicações clínicas, como o baixo peso. Dessa forma, preferimos classificála como um transtorno alimentar não especificado (TANE) ao invés de fobia específica. Essa confusão diagnóstica dificulta a pesquisa sobre a FA e o conhecimento de sua epidemiologia.

Assim como o recomendado para outros transtornos alimentares, o tratamento de L. foi interdisciplinar, tendo como pilar fundamental a reabilitação nutricional.

Uma das técnicas utilizadas pela terapia cognitivo-comportamental (TCC) é a exposição gradativa aos alimentos considerados proibidos, ou desencadeadores de náuseas e vômitos. Peluso et al. (1994) observaram melhora dos sintomas alimentares e do comportamento evitativo em uma paciente com FA, submetida a TCC, utilizando técnicas de dessensibilização sistemática e exposição. Singer et al. (1992) sugerem que a TCC consista de uma combinação individualizada de manejo de contingências, compartilhamento, dessensibilização, relaxamento e reestruturação cognitiva.

\section{CONCLUSÃO}

A FA é um transtorno psiquiátrico pouco reconhecido e comumente diagnosticado como AN em função da perda de peso acentuada. Dessa forma, é fundamental a investigação adequada dos diagnósticos diferenciais psiquiátricos em pacientes com desnutrição e baixo peso. Seu tratamento deve ser interdisciplinar, com ênfase na reabilitação nutricional e acompanhamento psicológico (sobretudo com as técnicas cognitivo-comportamentais). Além disso, deve ser implementado o mais breve possível, em função das complicações clínicas associadas.

A inclusão da FA como um diagnóstico psiquiátrico independente poderia facilitar o reconhecimento de suas características clínicas e epidemiológicas, assim como a avaliação de diferentes formas de tratamento.

\section{REFERÊNCIAS}

1. Manual diagnóstico e estatístico dos transtornos mentais. 4. ed. rev. (DSM-IV-TR). Porto Alegre: Artmed; 2002 
2. Higgs JF, Goodyer IM, Birch J. Anorexia nervosa and food avoidance emotional disorder. Arch Dis Child. 1989;64:346-351.

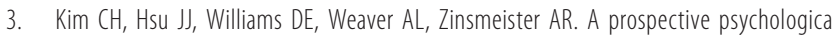
evaluation of patients with dysphagia of various etiologies. Dysphagia. 1996;11(1):34-40.

4. Shapiro J, Franko DL, Gagne A. Phagophobia: a form of psychogenic dysphagia. A new entity. Ann Otol Rhinol Laryngol. 1997;106(4):286-90.

5. Okada A, Tsukamoto C, Hosogi M, Yamanaka E, Watanabe K, Ootyou K, et al.. A study of psychopathology and treatment of children with phagophobia. Acta Med 0kayama. 2007;61(5):261-9.
6. McNally RJ. Chocking phobia: a reviw of literature. Compr Psychiatry. 1994;35(1):83-9.

7. Ciyiltepe M, Türkbay T. Phagophobia: a case report. Turk J Pediatr. 2006;48:80-84.

8. Peluso MAM, Neto FL. Fobia alimentar: relato de um caso clínico. J Bras Psiquiatr. 1994;43(10):527-31.

9. Singer LT, Ambuel B, Wade $S$, Jaffe AC. Cognitive-behavioral treatment of health-impairing food phobias in children. Case study. J Am Acad Child Adolesc Psychiatry. 1992; 31(5):847-52 